\title{
Distinguishing between Thymic Epithelial Tumors and Benign Cysts via Computed Tomography
}

Sang Hyup Lee, $M D^{1,2}$, Soon Ho Yoon, $M D^{1,2}$, Ju Gang Nam, $M D^{1,2}$, Hyung Jin Kim, $M D^{1,2}$, Su Yeon Ahn, $M D^{3,4}$, Hee Kyung Kim, $M D^{1,2}$, Hyun Ju Lee, $M D^{1,2}$, Hwan Hee Lee, $M D^{5}$, Gi Jeong Cheon, MD' ${ }^{5}$, Jin Mo Goo, MD ${ }^{1,2,6}$

${ }^{1}$ Department of Radiology, Seoul National University College of Medicine, Seoul, Korea; ${ }^{2}$ Department of Radiology, Seoul National University Hospital, Seoul, Korea; ${ }^{3}$ Department of Radiology, Konkuk University School of Medicine, Seoul, Korea; ${ }^{4}$ Department of Radiology, Konkuk University Medical Center, Konkuk University School of Medicine, Seoul, Korea; ${ }^{5}$ Department of Nuclear Medicine, Seoul National University College of Medicine, Seoul National University Hospital, Seoul, Korea; ${ }^{6}$ Institute of Radiation Medicine, Seoul National University Medical Research Center, Seoul, Korea

Objective: To investigate whether computed tomography (CT) and fluorine-18-labeled fluoro-2-deoxy-D-glucose (FDG) positron emission tomography (PET) may be applied to distinguish thymic epithelial tumors (TETs) from benign cysts in the anterior mediastinum.

Materials and Methods: We included 262 consecutive patients with pathologically proven TETs and benign cysts $5 \mathrm{~cm}$ or smaller who underwent preoperative CT scans. In addition to conventional morphological and ancillary CT findings, the relationship between the lesion and the adjacent mediastinal pleura was evaluated qualitatively and quantitatively. Mean lesion attenuation was measured on CT images. The maximum standardized uptake value (SUVmax) was obtained with FDGPET scans in 40 patients. CT predictors for TETs were identified with multivariate logistic regression analysis. For validation, we assessed the diagnostic accuracy and inter-observer agreement between four radiologists in a size-matched set of 24 cysts and 24 TETs using a receiver operating characteristic curve before and after being informed of the study findings. Results: The multivariate analysis showed that post-contrast attenuation of 60 Hounsfield unit or higher (odds ratio [OR], 12.734; 95\% confidence interval [CI], 2.506-64.705; $p=0.002)$ and the presence of protrusion from the mediastinal pleura (OR, 9.855; 95\% CI, 1.749-55.535; $p=0.009$ ) were the strongest CT predictors for TETs. SUVmax was significantly higher in TETs than in cysts $(5.3 \pm 2.4$ vs. $1.1 \pm 0.3 ; p<0.001)$. After being informed of the study findings, the readers' area under the curve improved from 0.872-0.955 to 0.949-0.999 ( $p=0.066-0.149)$. Inter-observer kappa values for protrusion were 0.630-0.941. Conclusion: Post-contrast CT attenuation, protrusion from the mediastinal pleura, and SUVmax were useful imaging features for distinguishing TETs from cysts in the anterior mediastinum.

Keywords: Mediastinum; Cystic mass; Thymic tumor; Computed tomography; FDG-PET/CT

\section{INTRODUCTION}

With the increasing use of low-dose chest computed

Received June 28, 2018; accepted after revision December 6, 2018.

Corresponding author: Soon Ho Yoon, MD, Department of Radiology, Seoul National University College of Medicine, 103 Daehak-ro, Jongno-gu, Seoul 03080, Korea.

- Tel: (822) 2072-2584 • Fax: (822) 743-7418

-E-mail:yshoka@gmail.com

This is an Open Access article distributed under the terms of the Creative Commons Attribution Non-Commercial License (https://creativecommons.org/licenses/by-nc/4.0) which permits unrestricted non-commercial use, distribution, and reproduction in any medium, provided the original work is properly cited. tomography (CT) screening for lung cancer, asymptomatic incidental lesions are frequently detected in the anterior mediastinum $(1,2)$. Such lesions generally originate from the thymus, and their long-axis diameter is usually $2 \mathrm{~cm}$ or less (median, $1.2 \mathrm{~cm}$; interquartile range, $0.9-1.6 \mathrm{~cm}$; range, $0.5-6.4 \mathrm{~cm}$ ) (2). Thymic epithelial tumors (TETs) are of primary concern for malignancy, but benign cysts account for many incidental benign lesions. Accordingly, TETs must be correctly distinguished from benign thymic cysts, when anterior mediastinal lesions are incidentally found.

On CT, incidental TETs typically present as round, solid lesions (2), while thymic benign cysts are traditionally recognized as having the $\mathrm{CT}$ attenuation of water $(\leq 20$ 
Hounsfield unit [HU]) (3). Water attenuation is a key differential imaging feature between cysts and TETs on CT scans. However, recent studies revealed that approximately three-fourths of thymic cysts showed hyper-attenuation to water $(4,5)$, potentially leading to misdiagnosis as TETs and unnecessary surgical resection $(6,7)$. Nevertheless, insufficient imaging features capable of distinguishing between TETs and benign cysts, other than water attenuation, have been identified on CT. In addition, although recent studies have suggested additional clinical value for fluorine18-labeled fluoro-2-deoxy-D-glucose (FDG) positron emission tomography (PET)/CT for predicting histological subtype of TETs, differentiation of benign cysts from small incidental TETs with PET/CT has not been examined $(8,9)$.

The purpose of this study was to investigate whether CT and ${ }^{18} \mathrm{~F}-\mathrm{FDG}$ PET findings may serve to distinguish TETs from benign cysts in the anterior mediastinum.

\section{MATERIALS AND METHODS}

\section{Study Population}

This retrospective study was approved by the Institutional Review Board and informed consent was waived. We applied the following inclusion criteria to the 385 consecutive patients who had undergone thymectomy between January 2007 and December 2014: 1) 18 years old or older; 2) availability of a preoperative chest CT scan; 3 ) final pathological diagnosis of either TET or benign cyst in the anterior mediastinum; and 4) long-axis lesion diameter of $5 \mathrm{~cm}$ or smaller, which encompassed $99 \%$ size range of incidental anterior mediastinal lesions in the general population (2). After excluding 113 cases with lesions larger than $5 \mathrm{~cm}, 262$ patients (mean age, 53.0 years; age range, 22-84 years; 135 men and 127 women) were included in this study (Fig. 1). The study group comprised 132 TETs (98 thymomas and 34 thymic carcinomas) and 130 benign cysts (112 thymic cysts and 18 bronchogenic cysts). The distribution of TET histological subtypes according to World Health Organization (WHO) classification is shown in Supplementary Table 1 in the online-only Data Supplement.

To validate the usefulness of differential CT features and to assess inter-observer agreement, we created an internal dataset of patients with pathologically proven TETs or benign cysts in the anterior mediastinum between January 2015 and December 2017 with the same criteria. Among the 24 thymic cysts and 73 TETs, we used a size-matched set of

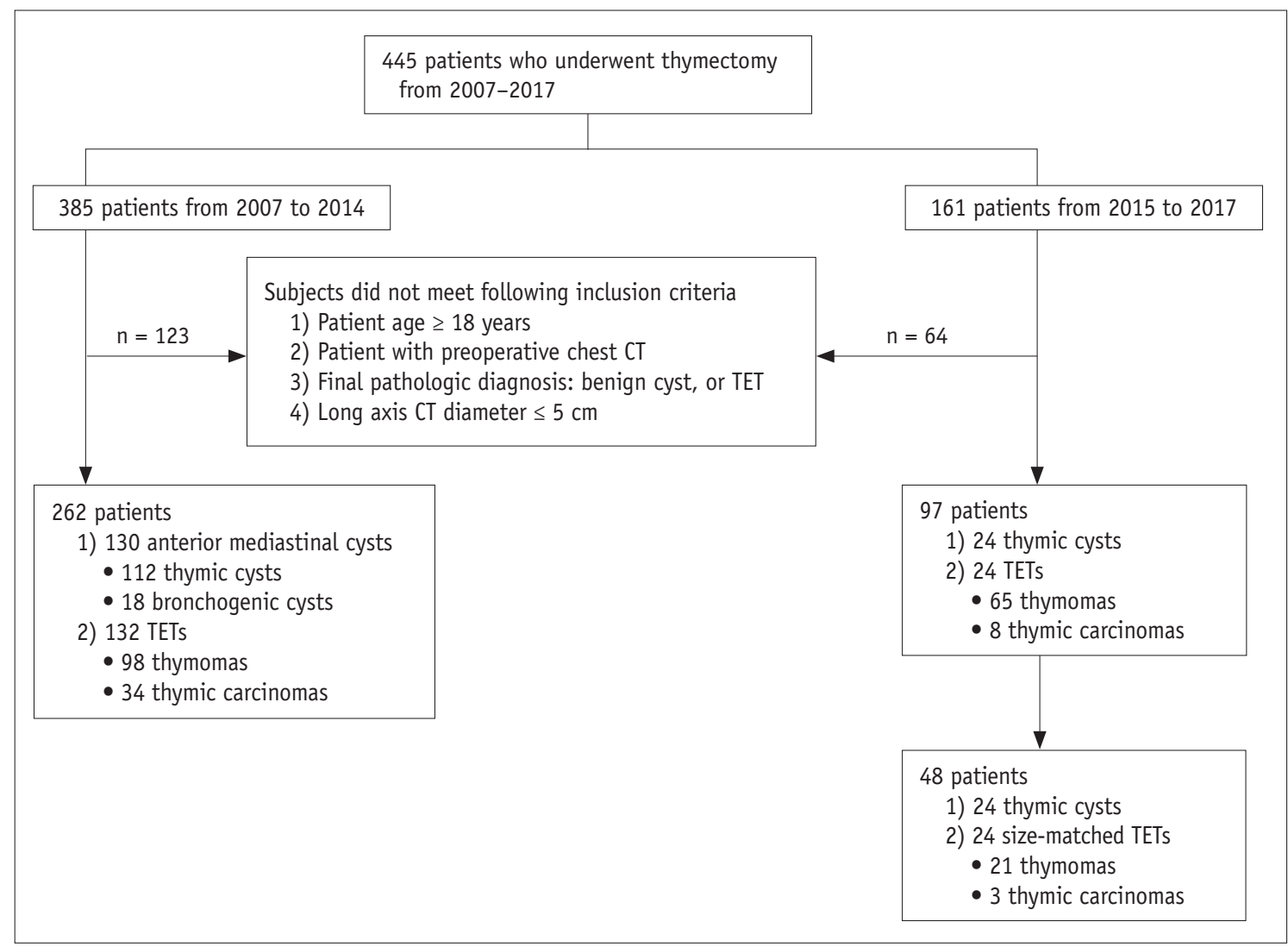

Fig. 1. Study diagram for patient inclusion. $C T=$ computed tomography, TET = thymic epithelial tumor 
24 cysts and 24 TETs for validation (mean age, 56.3 years; age range, $25-88$ years; 21 men and 27 women).

\section{Image Acquisition}

Chest CT examinations were performed using various multidetector scanners: an 8-channel scanner in 43 patients (Lightspeed Ultra, GE Healthcare, Milwaukee, WI, USA), a 16-channel scanner in 46 patients (Sensation 16, Siemens Healthineers, Erlangen, Germany), a 64-channel scanner in 141 patients (Brilliance 64, Philips Medical Systems, Cleveland, OH, USA; Discovery CT750 HD, GE Healthcare; Sensation 64, Siemens Healthineers), a 320-channel scanner in 11 patients (Aquilion One, Canon Medical Systems, Otawara, Japan), and a dual-source scanner in 21 patients (SOMATOM Definition, Siemens Healthineers). Among the 262 CT studies, 97 (37.0\%; 45 of 130 cysts and 52 of 132 TETs) had both pre- and post-contrast images; the remaining 165 studies had either pre-contrast $(n=59)$ or post-contrast images $(n=106)$. The detailed parameters for CT acquisition were as follows: tube voltage, $120 \mathrm{kVp}$; tube current, 120-250 mAs with automatic exposure control; slice thickness, $1.0-5.0 \mathrm{~mm}$; reconstruction interval, 0.6-5.0 mm; pitch, 0.9-1.0; rotation time, 0.5-1 second. Post-contrast CT images were obtained 60 seconds after intravenous administration of $90 \mathrm{~mL}$ iodinated contrast agent (iopamidol, $300 \mathrm{mg}$ iodine per milliliter) with an injection rate of $3.0 \mathrm{~mL} / \mathrm{s}$, followed by $30 \mathrm{~mL}$ saline chaser with the same rate.

\section{CT Image Analysis}

Two radiologists ( 2 and 12 years of experience in chest imaging, respectively) reviewed chest CT images on a picture archiving and communication system (INFINITT, Seoul, Korea) in a mediastinal window setting (width, $400 \mathrm{HU}$; level, $40 \mathrm{HU}$ ) to reach consensus. We analyzed conventional morphological features of the lesions and ancillary findings. The morphological features included the bi-dimensional longest long-axis and short-axis diameters, vertical location, contour, margin, shape, calcification, and gross fat; the ancillary findings encompassed adjacent organ invasion, pleural effusion, pericardial effusion, lymphadenopathy, satellite nodules, and distant metastasis. The vertical location was categorized as superior or inferior by comparing the epicenter of the lesion to the upper margin of the heart (10). The lesion contour was classified as smooth or lobulated. The margin was described as welldefined or ill-defined. Lymphadenopathy was considered present, if a mediastinal lymph node had a short-axis diameter of $1 \mathrm{~cm}$ or longer.

In addition, we evaluated the relationships between lesions and the adjacent mediastinal pleura qualitatively and quantitatively. First, the abutment to mediastinal pleura
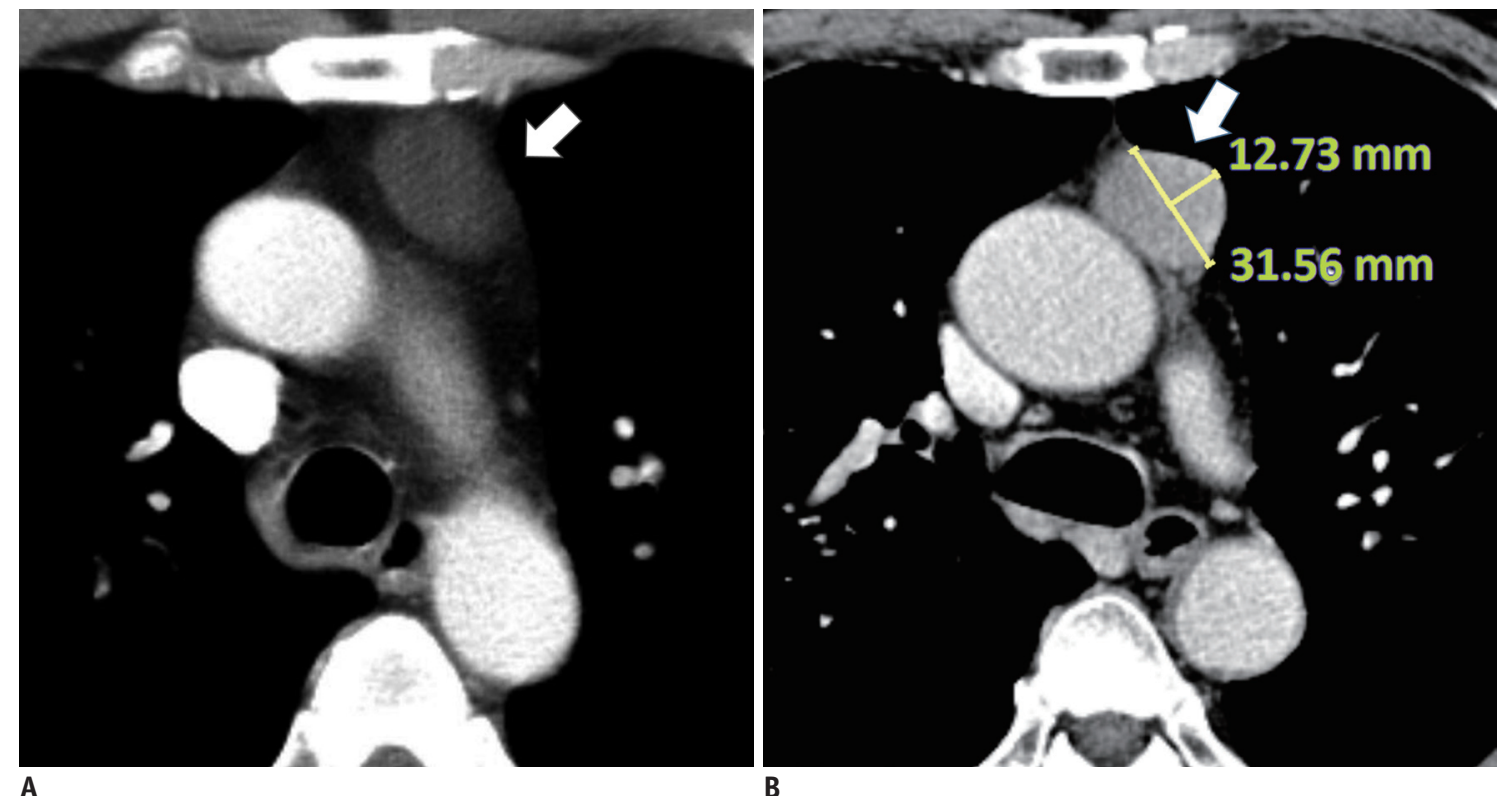

Fig. 2. Protrusion length and protrusion ratio on axial CT image.

A. 58-year-old female with 3.5-cm thymic cyst (arrow). Lesion abuts to adjacent mediastinal pleura but does not protrude: both protrusion length and protrusion ratio are 0. B. 64-year-old male with 3.4-cm thymic carcinoma (arrow), showing protrusion to mediastinal pleura. Protrusion length $=12.7 \mathrm{~mm}$, protrusion ratio $=(12.7 / 31.6) \times 100=40.2 \%$. 
was visually categorized as no abutment, abutment without protrusion, or abutment with protrusion towards the adjacent lung parenchyma. If a lesion touched the adjacent mediastinal pleura, the reviewers measured the abutment and protrusion lengths using an electronic ruler (Fig. 2). The protrusion length was defined as the perpendicular distance $(\mathrm{mm})$ from the abutted mediastinal pleura to the top of the apex protruding towards the adjacent lung parenchyma. The protrusion ratio was calculated as the protrusion length divided by the length of abutment to the mediastinal pleura. If a lesion did not touch the adjacent mediastinal pleura at all, the protrusion length and protrusion ratio were 0 .

One of the authors measured the mean CT attenuation values of the lesions. A circular region of interest (ROI) was placed in the largest possible homogeneously attenuating area. Measured attenuations were subtracted between post- and pre-contrast images to calculate the degree of absolute enhancement. Additional information including the adjacent major cardiovascular structure and the access route of intravenous contrast medium was collected for the 45 cysts with both pre- and post-contrast images. When the absolute enhancement value of a cyst was calculated to be $10 \mathrm{HU}$ or higher, it was considered as pseudoenhancement, which may be a consequence of beam-hardening effects from neighboring major vessels $(11,12)$.

\section{${ }^{18}$ F-FDG PET/CT Analysis}

Among the 262 patients, 49 underwent a preoperative ${ }^{18} \mathrm{~F}-\mathrm{FDG}$ PET/CT scan. A standardized uptake value (SUV) was not obtainable for 9 patients with PET images acquired in other institutions. Accordingly, we analyzed PET/CT images for 40 lesions (12 thymic cysts and 28 TETs, consisting of 17 thymomas and 11 thymic carcinomas). PET/CT scans were performed on an integrated PET/CT scanner (Biograph Truepoint or mCT40, Siemens Healthineers, Knoxville, TN, USA). After fasting for $\geq 6$ hours, patients were injected with ${ }^{18} \mathrm{~F}-\mathrm{FDG}(0.14 \mathrm{mCi} / \mathrm{kg})$, and images were acquired 1 hour later. Serum glucose concentrations were measured before ${ }^{18} \mathrm{~F}-\mathrm{FDG}$ injection and were required to be $<200$ $\mathrm{mg} / \mathrm{dL}$. A CT scan (40 mA and $120 \mathrm{kVp}$ ) was performed for attenuation correction without contrast enhancement prior to PET scanning. CT scans were acquired using a 3-mm section thickness from the skull base to the mid-thigh; images were reconstructed using a $512 \times 512$ matrix and a $50-\mathrm{cm}$ field of view. PET scans obtained from the midthigh to the skull base were reconstructed with a $200 \mathrm{x}$
200 matrix using the ordered subset expectation maximum iterative reconstruction algorithm, a 5-mm Gaussian filter, and a 78-cm field of view. A nuclear radiologist (with 4 years of clinical experience in nuclear medicine) measured the maximum SUV (SUVmax) of the lesion, correcting for body weight.

\section{Validation of CT Image Interpretation}

CT images of 24 thymic cysts (mean long-axis diameter, $2.3 \pm 0.9 \mathrm{~cm}$ ) and 24 size-matched TETs (mean long-axis diameter, $2.3 \pm 0.9 \mathrm{~cm}$ ) were independently evaluated in random order by four radiologists $(22,6,5$, and 5 years of experience in chest imaging, respectively), blinded to pathological results. For the first session, each reader was asked to identify each lesion using the following 5-point scale: 1 , thymic cyst, most likely; 2, cyst, more likely than tumor; 3, equivocal; 4, tumor, more likely than cyst; and 5, tumor, most likely. For the second session, the readers were informed of the results of the training dataset, and subsequently asked to measure the CT attenuation of ROI in the pre- and post-contrast images, the abutment length, protrusion length, and protrusion ratio of each lesion, and to determine the presence of protrusion. The 5-point scale was assessed again based on the newly obtained information and measured data. To reduce recall bias, an interval of more than 4 weeks separated the interpretation sessions.

\section{Statistical Analysis}

Categorical variables were compared using the chisquare or Fisher exact test, and the independent $t$ test was performed for continuous variables. Fisher's exact test was performed to identify any anatomical factors related to pseudoenhancement of benign cysts. To determine the optimal cut-off for continuous variables, we used receiver operating characteristic (ROC) curve analysis for the following parameters: protrusion length, protrusion ratio, mean attenuation value in pre- and post-contrast images, degree of absolute enhancement, and SUVmax. The diagnostic capacity of SUVmax was compared with that of protrusion length and protrusion ratio, respectively, using the DeLong test. CT attenuation variables were not included in the DeLong test due to the small number of patients who underwent both PET/CT and contrast enhanced CT scan $(n=7)$.

Univariate and multivariate logistic regression analyses were performed to identify significant CT predictors for TETS (13), and additional multivariate logistic regression analysis was performed between the low risk group (WHO types A, 
Table 1. CT and ${ }^{18}$ F-FDG PET Findings of Benign Cysts And TETs in Anterior Mediastinum

\begin{tabular}{|c|c|c|c|}
\hline $\mathrm{CT}$ and ${ }^{18} \mathrm{~F}-\mathrm{FDG}$ PET Findings & TETs $(n=132)$ & Benign Cysts $(n=130)$ & $P$ \\
\hline \multicolumn{4}{|l|}{ Morphologic findings } \\
\hline Long-axis diameter $(\mathrm{cm})$ & $3.4 \pm 1.0$ & $2.1 \pm 0.9$ & $<0.001$ \\
\hline Short-axis diameter $(\mathrm{cm})$ & $2.6 \pm 3.5$ & $1.5 \pm 0.6$ & $<0.001$ \\
\hline Superior location above heart (vs. inferior location) (\%) & $88(116)$ & $96(125)$ & 0.021 \\
\hline Lobulating contour (vs. smooth) (\%) & $63(83)$ & $17(22)$ & $<0.001$ \\
\hline Ill-defined margin (vs. well-defined) (\%) & $20(27)$ & $6(8)$ & 0.001 \\
\hline Oval/irregular shape (vs. round) (\%) & $76(100)$ & $69(90)$ & 0.238 \\
\hline Calcification (\%) & $11(14)$ & $2(3)$ & 0.010 \\
\hline Gross fat & 0 & 0 & N/A \\
\hline \multicolumn{4}{|l|}{ Ancillary findings (\%) } \\
\hline Adjacent organ invasion & $7(9)$ & 0 & 0.003 \\
\hline Pleural effusion & $2(3)$ & 0 & 0.247 \\
\hline Pericardial effusion & 0 & 0 & N/A \\
\hline Lymphadenopathy & $4(5)$ & 0 & 0.060 \\
\hline Satellite nodule & $5(6)$ & $2(3)$ & 0.500 \\
\hline Metastasis & $6(8)$ & 0 & $<0.001$ \\
\hline \multicolumn{4}{|l|}{ Relationship between lesion and adjacent mediastinal pleura } \\
\hline \multicolumn{4}{|l|}{ Visual categorization (\%) } \\
\hline No abutment & $6(8)$ & $20(26)$ & \multirow{3}{*}{$<0.001$} \\
\hline Abutment & $11(15)$ & $67(88)$ & \\
\hline Protrusion & $83(109)$ & $13(16)$ & \\
\hline Protrusion length (mm) & $10.0 \pm 8.7$ & $0.8 \pm 2.9$ & $<0.001$ \\
\hline Protrusion ratio (\%) & $32.3 \pm 33.0$ & $3.0 \pm 8.9$ & $<0.001$ \\
\hline CT attenuation (HU) & $(n=52)$ & $(n=45)$ & \\
\hline Pre-contrast CT attenuation & $47.3 \pm 12.1$ & $31.4 \pm 18.0$ & $<0.001$ \\
\hline Post-contrast CT attenuation & $88.8 \pm 28.0$ & $44.1 \pm 21.9$ & $<0.001$ \\
\hline Absolute enhancement & $41.5 \pm 27.8$ & $12.8 \pm 21.4$ & $<0.001$ \\
\hline${ }^{18} \mathrm{~F}-\mathrm{FDG}$ PET/CT & $(n=28)$ & $(n=12)$ & \\
\hline SUVmax & $5.3 \pm 2.4$ & $1.1 \pm 0.3$ & $<0.001$ \\
\hline
\end{tabular}

Data in parenthesis indicate number of patients. $\mathrm{CT}=$ computed tomography, $\mathrm{HU}=$ Hounsfield unit, N/A = not applicable, PET = positron emission tomography, SUVmax = maximum standardized uptake value, TET = thymic epithelial tumor, ${ }^{18} \mathrm{~F}-\mathrm{FDG}=$ fluorine- 18 -labeled fluoro2-deoxy-D-glucose

$A B$, and $B 1)$ and cysts.

In the validation dataset, the areas under the ROC curves (AUC) of the two interpretation sessions for each reader were compared using the DeLong test to check whether awareness of the results of the training dataset improved readers' diagnostic performance. Kappa statistics and intraclass correlation coefficients (ICCS) were used to measure inter-observer agreement between radiologists in the validation dataset, with values of $0.00-0.20,0.21-0.40$, $0.41-0.60,0.61-0.80$, and $0.81-1.00$ considered as poor, fair, moderate, good, and excellent, respectively. The $95 \%$ limits of agreement between paired readers were obtained using Bland-Altman analysis.

All statistical analyses were performed using MedCalc for Windows, version 15.0 (MedCalc Software, 0stend, Belgium). $P$ values $<0.05$ were considered to indicate a statistically

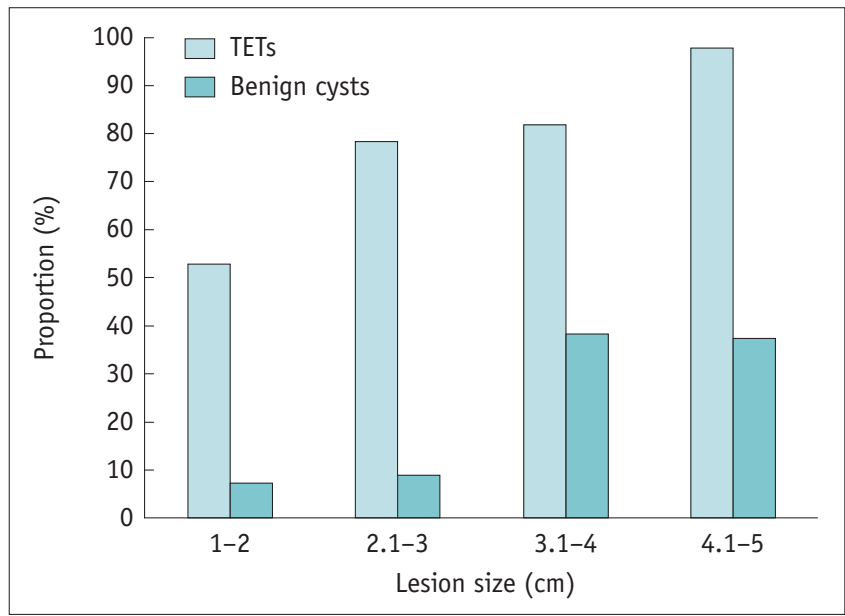

Fig. 3. Proportion of TETs and benign cysts that showed protrusion from adjacent mediastinal pleura according to lesion size. No TET was smaller than $1 \mathrm{~cm}$. Protrusion was absent in 9 thymic cysts smaller than $1 \mathrm{~cm}$. 
significant difference.

\section{RESULTS}

The univariate analysis (Table 1) showed that TETs were significantly larger than benign cysts (mean long-axis diameter, $3.4 \mathrm{~cm}$ vs. $2.1 \mathrm{~cm} ; p<0.001$ ). TETs were more likely than cysts to be located below the upper border of the heart $(p=0.021)$, to have a lobulated contour ( $p<$ $0.001)$, to have an ill-defined margin $(p=0.001)$, and to show calcification $(p=0.010)$. Direct invasion of an adjacent organ $(p=0.003)$, pleural effusion $(p=0.247)$, lymphadenopathy $(p=0.060)$, and distant metastasis $(p<0.001)$ were exclusively observed in TETs. TETs more frequently protruded from the adjacent mediastinal pleura than benign cysts ( $83 \%$ vs. $13 \% ; p<0.001)$, and showed

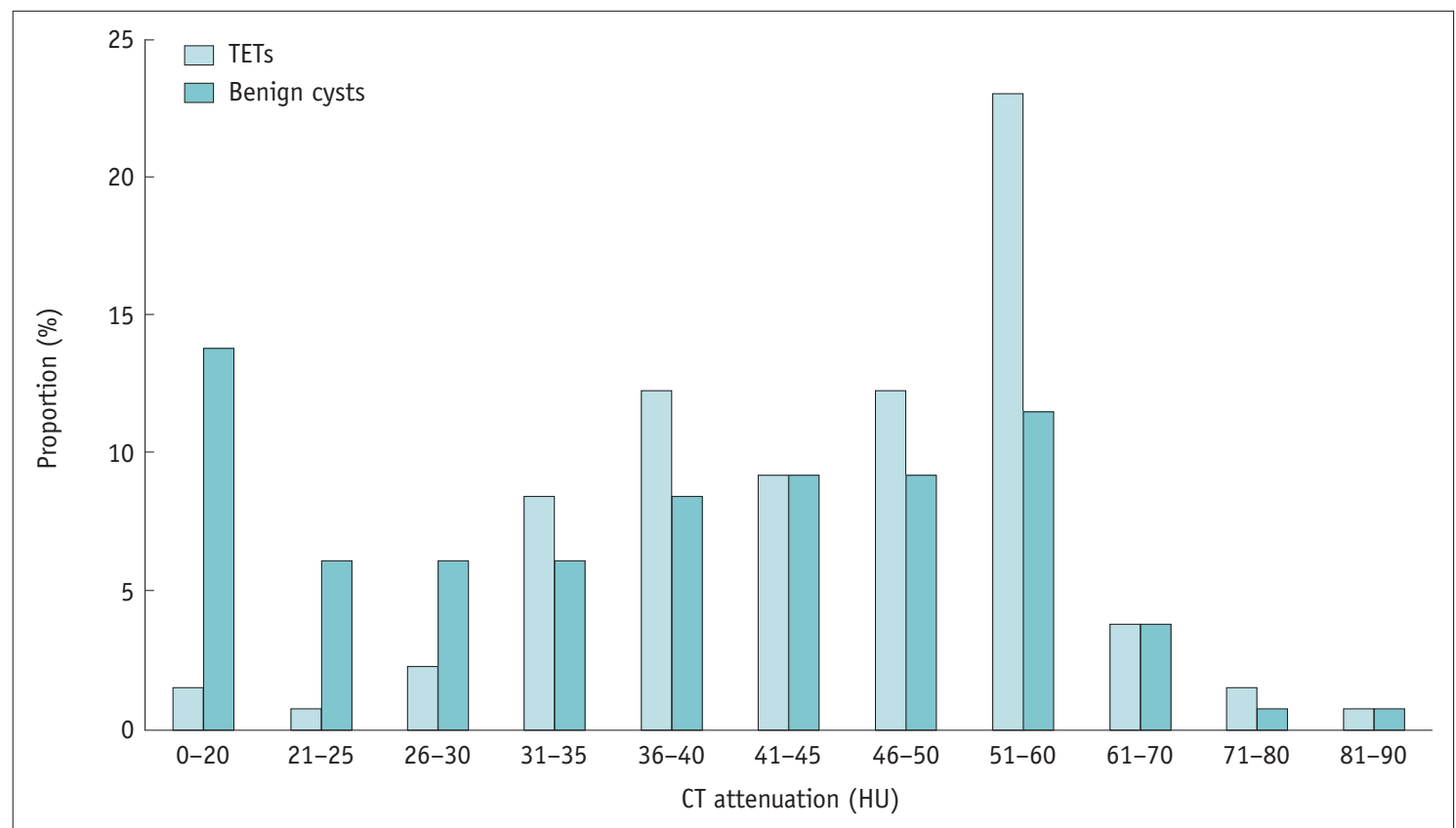

A

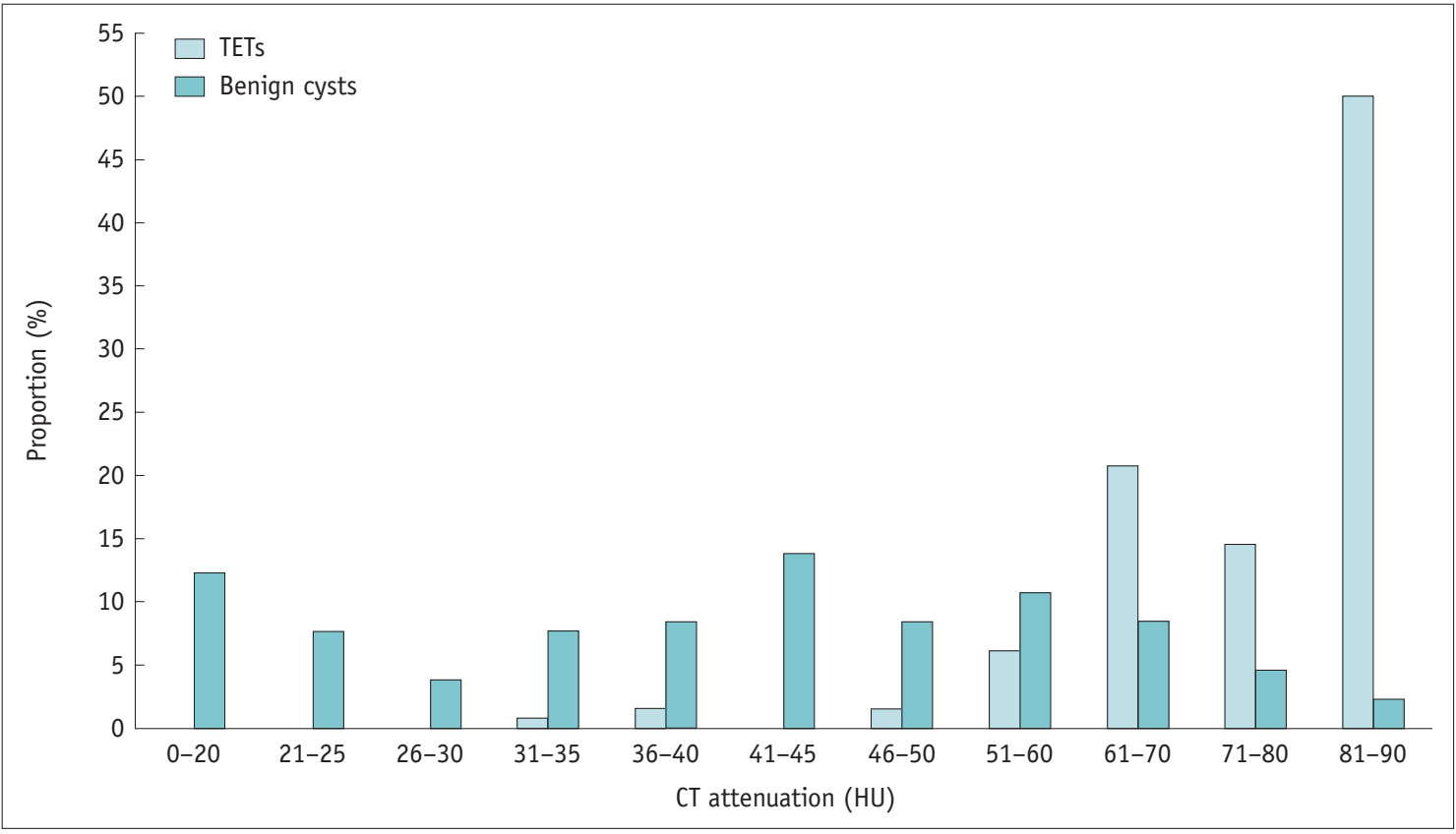

B

Fig. 4. Distribution of (A) pre- and (B) post-contrast CT attenuation of TETs and benign cysts. $\mathrm{HU}=$ Hounsfield unit 
greater length $(10.0 \mathrm{~mm}$ vs. $0.8 \mathrm{~mm} ; p<0.001)$ and larger protrusion ratio $(32.3 \%$ vs. $3.0 \% ; p<0.001)$. The presence of protrusion depended on lesion size (Fig. 3).
The mean CT attenuation of benign cysts was higher than water attenuation in pre-contrast $(31.4 \pm 18.0 \mathrm{HU})$ and post-contrast images $(44.1 \pm 21.9 \mathrm{HU})$, and was

Table 2. Results of Subgroup Analysis on Benign Cysts according to Presence of Pseudoenhancement

\begin{tabular}{|c|c|c|c|}
\hline CT Findings & $\begin{array}{l}\text { Pseudoenhancement }(+)^{*} \\
(42 \%, \mathrm{n}=19)\end{array}$ & $\begin{array}{l}\text { Pseudoenhancement (-) } \\
(58 \%, \mathrm{n}=26)\end{array}$ & $P$ \\
\hline Pre-contrast $\mathrm{CT}$ attenuation (HU) & $22.1 \pm 18.5$ & $38.2 \pm 15.0$ & 0.003 \\
\hline Post-contrast $\mathrm{CT}$ attenuation (HU) & $54.6 \pm 25.4$ & $36.4 \pm 15.9$ & 0.005 \\
\hline Absolute enhancement (HU) & $32.5 \pm 18.0$ & $-1.7 \pm 8.7$ & $<0.001$ \\
\hline Long-axis diameter $(\mathrm{cm})$ & $2.0 \pm 0.8$ & $2.1 \pm 0.8$ & 0.724 \\
\hline Presence of protrusion (\%) & $16(n=3)$ & $4(n=1)$ & 0.295 \\
\hline Adjacent Major Cardiovascular Structure & \multicolumn{2}{|c|}{ Intravenous Contrast Injection Route (Rt./Lt. Upper Extremity) } & $P$ (Rt. vs. Lt.) \\
\hline Left innominate vein $(n=9)$ & $0 / 4$ & $4 / 1$ & 0.048 \\
\hline Aorta $(n=31)$ & $4 / 9$ & $12 / 6$ & 0.073 \\
\hline Main pulmonary artery $(n=4)$ & $1 / 0$ & $3 / 0$ & $\mathrm{~N} / \mathrm{A}$ \\
\hline Right ventricle $(n=1)$ & $1 / 0$ & $0 / 0$ & $\mathrm{~N} / \mathrm{A}$ \\
\hline
\end{tabular}

*Pseudoenhancement was considered as positive for lesions with absolute enhancement $\geq 10 \mathrm{HU}$. Lt. = left, Rt. = right

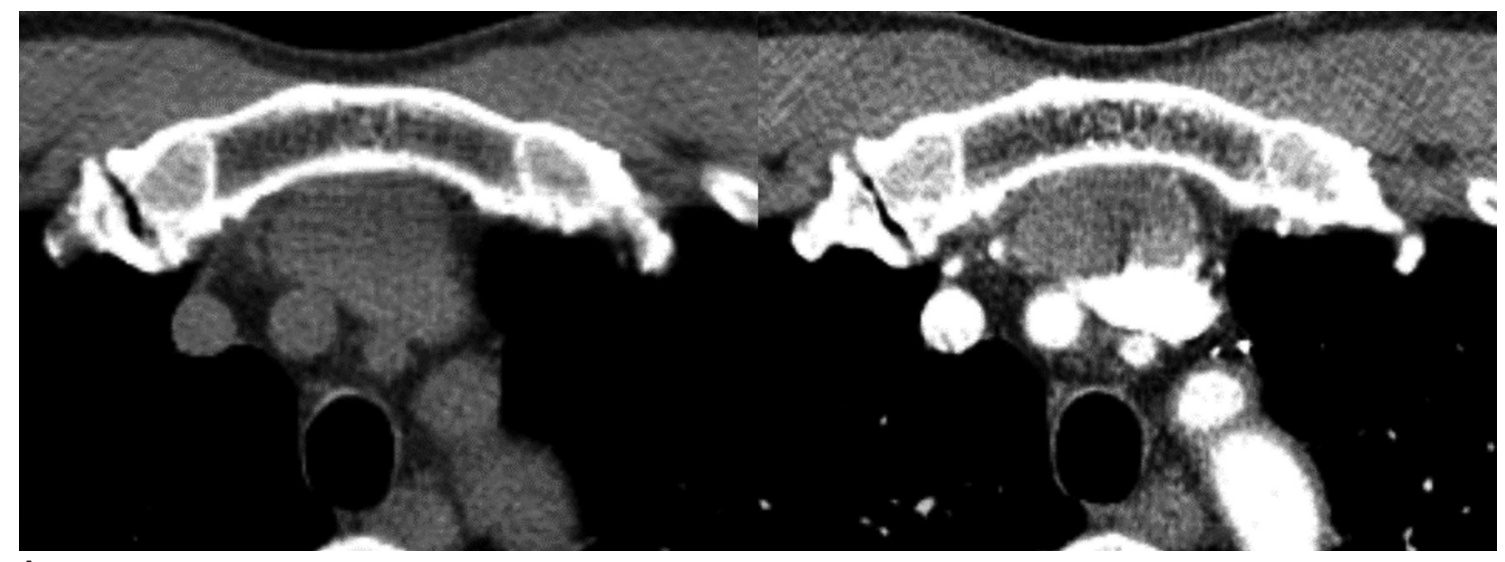

A

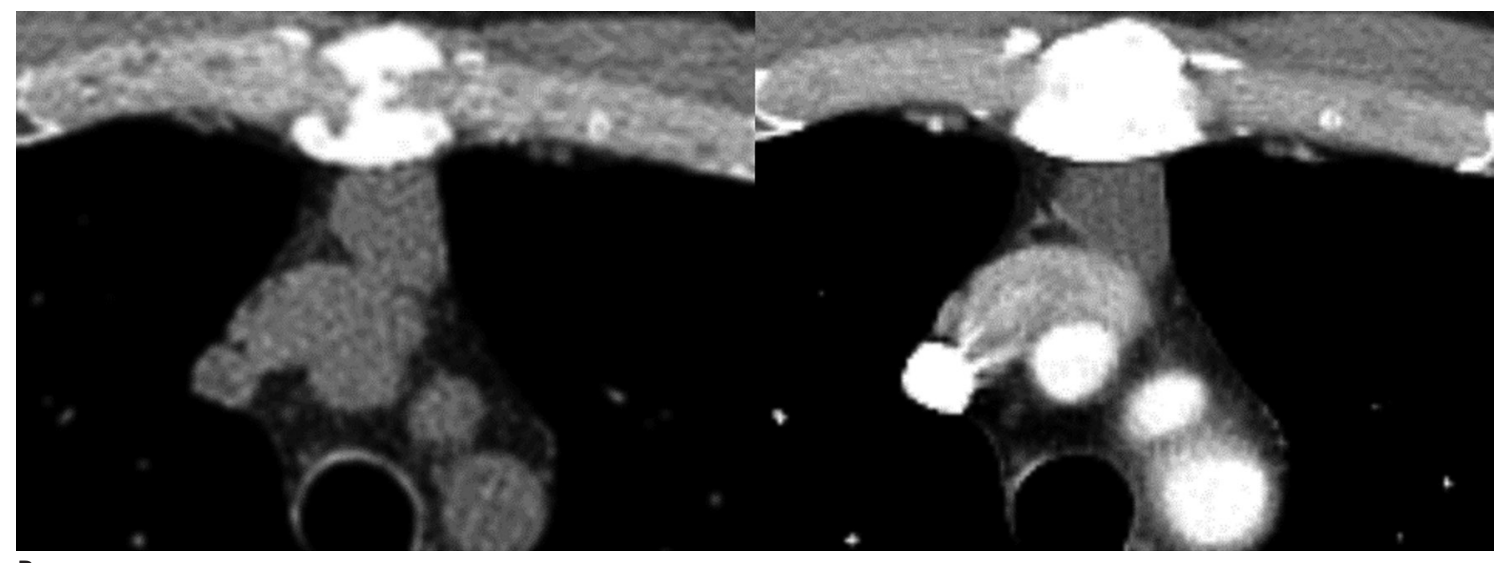

B

Fig. 5. Pseudoenhancement in thymic cysts.

A. 61-year-old male with 3.5-cm thymic cyst showing pseudoenhancement. Lesion is located at prevascular space, abutting left innominate vein. Pre-contrast study (left) shows round shaped, smooth-margined mass with internal fluid attenuation. After contrast enhancement (right), beamhardening artifacts from left innominate vein with injected dense contrast medium create bright streaky high attenuations on mass, causing pseudoenhancement. Mean CT attenuation values measured at two phases were $14 \mathrm{HU}$ and $49 \mathrm{HU}$, respectively, resulting in absolute enhancement of $35 \mathrm{HU}$. B. 52-year-old male with 2.2-cm thymic cyst without enhancement. Lesion is also abutting left innominate vein, but contrast medium is being injected through right upper extremity vein. Mean CT attenuation values measured at two phases were $44 \mathrm{HU}$ and $42 \mathrm{HU}$, respectively. 
significantly lower than that of TETs (pre-contrast, $47.3 \pm$ $12.1 \mathrm{HU}$; post-contrast, $88.8 \pm 28.0 \mathrm{HU}$; each $p<0.001$ ) (Table 1, Fig. 4). The mean absolute enhancement of benign cysts was slightly higher than $10 \mathrm{HU}(12.8 \pm 21.4 \mathrm{HU})$ and was significantly lower than that of TETs $(41.5 \pm 27.8$ HU) $(p<0.001)$. However, 19 of the 45 benign cysts had distinct pseudoenhancement of $10 \mathrm{HU}$ or more on pre- and post-contrast images $(32.5 \pm 18.0 \mathrm{HU}$; range, 10.1-66.2 $\mathrm{HU})$. Intravenous contrast medium injection through the left upper extremity was a significant factor for inducing pseudoenhancement at the cysts adjacent to the left innominate vein $(p=0.048)$ (Table 2, Fig. 5). PET/CT scans showed that mean SUVmax was significantly higher in TETs than in cysts, without any overlap ( $5.3 \pm 2.4$ vs. $1.1 \pm 0.3$; $p<0.001$ ) (Table 1). The optimal threshold values for each continuous parameter obtained from ROC curve analysis are shown in Table 3. The DeLong test revealed that SUVmax has better diagnostic capacity than protrusion length and ratio ( $p=0.034$ for both protrusion length and ratio).

Multivariate logistic regression analysis adjusting for lesion size showed that post-contrast attenuation of 60 $\mathrm{HU}$ or higher (odds ratio [OR], 12.734; 95\% confidence interval $[\mathrm{CI}], 2.506-64.705 ; p=0.002)$ and the presence of protrusion from the mediastinal pleura $(0 R, 9.855 ; 95 \%$ CI, $1.749-55.535 ; p=0.009$ ) were significant independent predictors for TETs (Table 4). In the multivariate logistic regression analysis using the protrusion ratio instead of the presence of protrusion, similar results were found for a post-contrast attenuation of $\geq 60 \mathrm{HU}(0 \mathrm{R}, 13.145 ; 95 \% \mathrm{CI}$, $2.602-66.417 ; p=0.002)$ and a protrusion ratio of $\geq 13 \%$ $(0 R, 9.346 ; 95 \%$ CI, 1.638-53.318; $p=0.012)$ (Table 4). Those findings were also significant independent predictors for discrimination of low-risk thymomas from cysts (Supplementary Table 2-1 and 2-2 in the online-only Data Supplement).

The ROC curve comparisons between the validation sessions for each reader are shown in Figure 6 . The AUC values of all four readers increased from 0.872-0.955 to $0.949-0.999$ in the second session, although the differences were not statistically significant ( $p=0.066$ 0.149 ), possibly due to high diagnostic accuracy of the 1st validation session and the relatively small size of the internal dataset.

The inter-observer agreement for the presence of abutment and protrusion was moderate to excellent $(\kappa=$ $0.404-0.941)$ and good to excellent $(\kappa=0.630-0.941)$,

Table 3. Optimal Threshold Values for Each Continuous Parameter Obtained from Receiver Operating Characteristic Curve Analysis

\begin{tabular}{lcccc}
\hline \multicolumn{1}{c}{ Parameters } & Optimal Threshold Value & Area Under Curve & Sensitivity (\%) & Specificity (\%) \\
\hline Protrusion length (mm) & 1.7 & 0.875 & 83 & 89 \\
Protrusion ratio (\%) & 13 & 0.879 & 81 & 89 \\
Pre-contrast attenuation (HU) & 35 & 0.756 & 83 & 58 \\
Post-contrast attenuation (HU) & 60 & 0.901 & 87 & 83 \\
Absolute enhancement (HU) & 17 & 0.805 & 100 & 71 \\
SUVmax & 1.43 & 1.000 & & 100 \\
\hline
\end{tabular}

Table 4. Results of Multivariate Logistic Regression Analysis including Either (a) Presence of Protrusion or (b) Protrusion Ratio

\begin{tabular}{|c|c|c|c|}
\hline CT Findings & Odds Ratio & $95 \% \mathrm{CI}$ & $P$ \\
\hline \multicolumn{4}{|l|}{$(a)^{*}$} \\
\hline Long-axis diameter $(\mathrm{cm})$ & 2.573 & $0.991-6.682$ & 0.052 \\
\hline Presence of protrusion & 9.855 & $1.749-55.535$ & 0.009 \\
\hline Lobulated contour & 5.598 & $0.761-41.194$ & 0.091 \\
\hline Pre-contrast attenuation $\geq 35 \mathrm{HU}$ & 4.808 & $0.654-35.337$ & 0.123 \\
\hline Post-contrast attenuation $\geq 60 \mathrm{HU}$ & 12.734 & $2.506-64.705$ & 0.002 \\
\hline \multicolumn{4}{|l|}{$(b)^{*}$} \\
\hline Long-axis diameter $(\mathrm{cm})$ & 2.574 & $0.983-6.743$ & 0.054 \\
\hline Protrusion ratio $\geq 13 \%$ & 9.346 & $1.638-53.318$ & 0.012 \\
\hline Lobulated contour & 6.449 & $0.927-44.858$ & 0.060 \\
\hline Pre-contrast attenuation $\geq 35 \mathrm{HU}$ & 5.329 & $0.731-38.837$ & 0.099 \\
\hline Post-contrast attenuation $\geq 60 \mathrm{HU}$ & 13.145 & $2.602-66.417$ & 0.002 \\
\hline
\end{tabular}

*0ther variables were removed from analysis using backward enter model, in which variables that show low significance level with $p$ value larger than 0.1 are removed from regression model. CI = confidence interval 
respectively (Table 5). The inter-observer agreement for quantitative values of the lesions was excellent (ICC $=$ 0.824-0.908), without showing significant measurement bias between the readers in Bland-Altman analysis. The ICCs and the 95\% limits of agreement are summarized in Table 6 .

\section{DISCUSSION}

Our study found that post-contrast attenuation of $\geq$ $60 \mathrm{HU}$ and either the presence of protrusion from the mediastinal pleura or a protrusion ratio of $\geq 13 \%$ were the
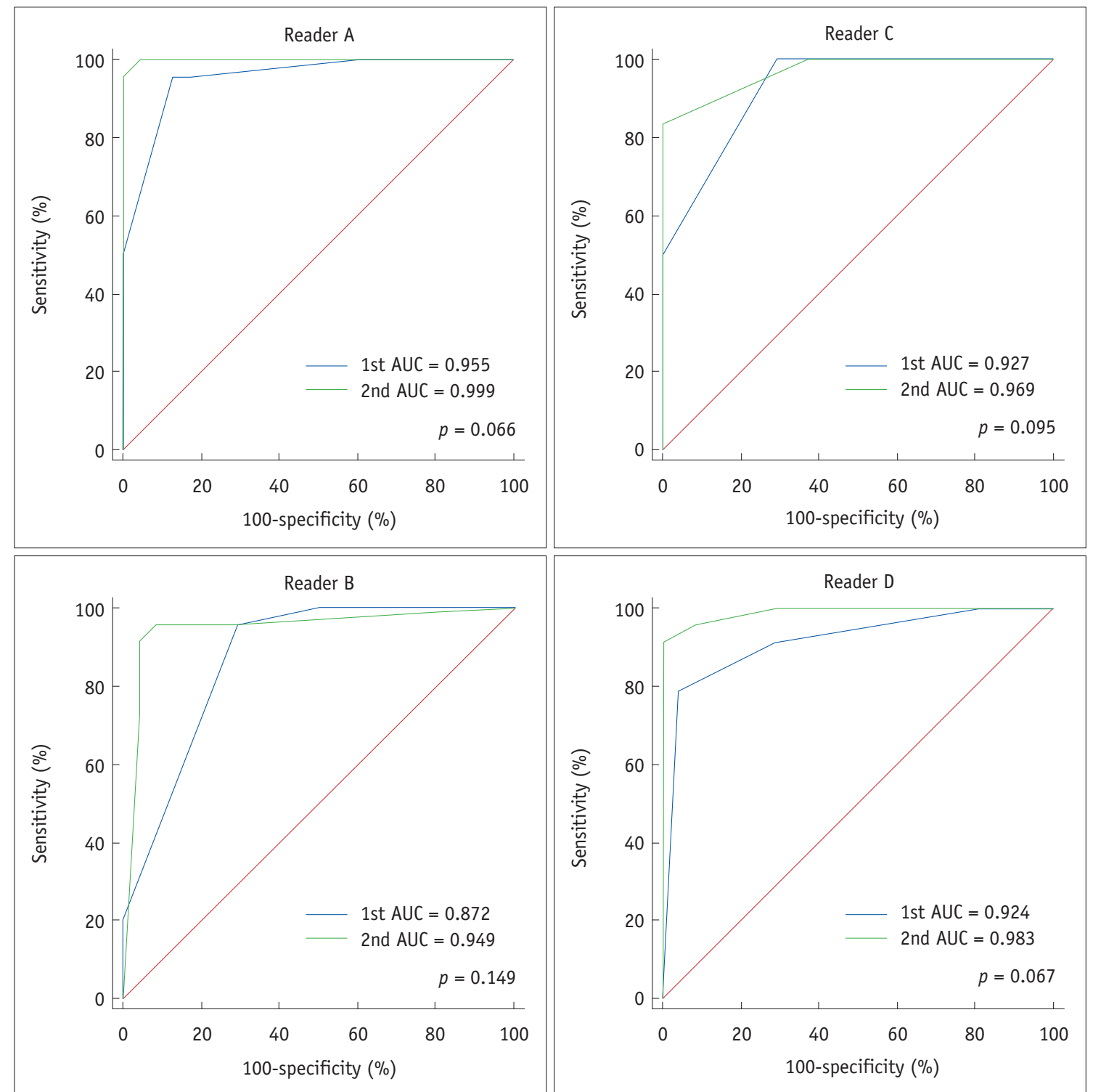

Fig. 6. ROC curves of four radiologists before and after instruction of study results. $A U C=$ areas under $R O C$ curve, $R O C=$ receiver operating characteristic

Table 5. Inter-Observer Agreement in Assessing Presence of Abutment and Protrusion to Mediastinal Pleura

\begin{tabular}{rccccccc}
\hline \multirow{2}{*}{ k value } & $\begin{array}{c}\text { Reader } 1 \text { vs. } \\
\text { Reader } 2\end{array}$ & $\begin{array}{c}\text { Reader } 1 \text { vs. } \\
\text { Reader } 3\end{array}$ & $\begin{array}{c}\text { Reader } 1 \text { vs. } \\
\text { Reader } 4\end{array}$ & $\begin{array}{c}\text { Reader } 2 \text { vs. } \\
\text { Reader } 3\end{array}$ & $\begin{array}{c}\text { Reader } 2 \text { vs. } \\
\text { Reader } 4\end{array}$ & $\begin{array}{c}\text { Reader } 3 \text { vs. } \\
\text { Reader } 4\end{array}$ \\
\hline \multirow{2}{*}{ Abutment } & 0.523 & 0.478 & 0.893 & 0.941 & 0.444 & 0.404 \\
Protrusion & $(0.244-0.802)$ & $(0.206-0.750)$ & $(0.687-1.000)$ & $(0.827-1.000)$ & $(0.162-0.726)$ & $(0.134-0.675)$ \\
& 0.767 & 0.827 & 0.700 & 0.941 & 0.683 & 0.630 \\
& $(0.553-0.981)$ & $(0.641-1.000)$ & $(0.467-0.933)$ & $(0.827-1.000)$ & $(0.431-0.935)$ & $(0.370-0.891)$ \\
\hline
\end{tabular}

Data in parenthesis indicates $95 \% \mathrm{CI}$. 
Table 6. ICC and Bland-Altman Analysis of Quantitative Measurements Across Four Readers

\begin{tabular}{|c|c|c|c|}
\hline CT Findings & ICC & Lower End of $95 \% \mathrm{CI}$ & Upper End of $95 \%$ CI \\
\hline Pre-contrast $\mathrm{CT}$ attenuation (HU) & 0.824 & 0.696 & 0.915 \\
\hline Post-contrast $\mathrm{CT}$ attenuation (HU) & 0.908 & 0.853 & 0.948 \\
\hline Abutment length (mm) & 0.828 & 0.736 & 0.899 \\
\hline Protrusion length (mm) & 0.870 & 0.797 & 0.924 \\
\hline Protrusion ratio $(\%)$ & 0.856 & 0.776 & 0.916 \\
\hline Bland-Altman Analysis & Mean Difference & Lower End of $95 \%$ Limit of Agreement & Upper End of $95 \%$ Limit of Agreement \\
\hline Pre-contrast $\mathrm{CT}$ attenuation (HU) & $-1.2 \pm 0.8$ & $-20.5 \pm 4.3$ & $18.2 \pm 4.0$ \\
\hline Post-contrast $\mathrm{CT}$ attenuation (HU) & $-0.2 \pm 5.6$ & $-26.9 \pm 6.6$ & $26.6 \pm 6.6$ \\
\hline Abutment length (mm) & $-0.7 \pm 4.7$ & $-17.6 \pm 9.1$ & $16.3 \pm 4.8$ \\
\hline Protrusion length (mm) & $-0.5 \pm 0.5$ & $-6.6 \pm 2.4$ & $5.7 \pm 1.5$ \\
\hline Protrusion ratio $(\%)$ & $-1.0 \pm 0.6$ & $-21.3 \pm 3.7$ & $18.7 \pm 4.1$ \\
\hline
\end{tabular}

$\mathrm{ICC}=$ intraclass correlation coefficient

strongest CT predictors for TETs. SUVmax $\geq 1.43$ served as an exclusive PET predictor for TETs. Being informed of those CT predictors improved readers' performance in distinguishing TETs from benign cysts in the validation dataset, and CT predictors were reasonably well reproduced across readers.

Unlike other simple cysts usually with pure water density on $\mathrm{CT}$, thymic cysts may appear with higher $\mathrm{CT}$ attenuation than serous fluid due to their protein-rich content or hemorrhage $(4,5,14)$. Previous studies reported that the mean pre-contrast CT value of thymic cysts was $22.9 \mathrm{HU}$ (range, $-19.9-58.2 \mathrm{HU}$ ) (7), and that $38.0 \%$ of thymic cysts showed higher CT values than water attenuation (> $20 \mathrm{HU}$ ) (15). In our study, the mean pre-contrast CT value for cysts was $31 \mathrm{HU}$, which is consistent with the findings of previous studies. Although the mean pre-contrast CT value for TETs was higher (47 HU), there was no significant between-group difference in the multivariate analysis.

The mean post-contrast CT value of cysts was $44 \mathrm{HU}$, showing a mean absolute enhancement of $13 \mathrm{HU}$ compared to the pre-contrast phase. Classically, benign simple cysts do not show contrast enhancement. According to Araki et al. (5), the pre-contrast mean CT value for 11 thymic cysts was $45 \mathrm{HU}$, whereas the enhanced mean $\mathrm{CT}$ value was $38 \mathrm{HU}$, indicating a lack of enhancement. However, in our study, a larger sample (45 cysts) was analyzed and many of our cases showed enhancement mimicking a solid lesion. This can result from pseudoenhancement due to the overlapping beam-hardening effect (11), when the cyst is adjacent to the dense contrast medium passing through mediastinal great vessels (Fig. 5). Pseudoenhancement can cause misdiagnosis of cysts as solid lesions, and this similar phenomenon is widely observed in renal cysts (11, $12,16-18)$. Our results showed that contrast medium injection though the left upper extremity vein increased the chance of pseudoenhancement in cysts adjacent to the left innominate vein (Table 2, Fig. 5). Unlike the right side, the left innominate vein courses through the anterior mediastinum, crosses the midline and thereby there is a higher chance that it is in close contact with a mediastinal lesion.

TETs showed higher mean CT values (89 HU) than cysts on contrast-enhanced CT examinations. ROC analysis revealed an excellent diagnostic performance, with an AUC value of 0.901 . The optimal cutoff value was $60 \mathrm{HU}$, which could distinguish between cysts and TETs with a sensitivity of $86.5 \%$ and a specificity of $82.2 \%$. It should be noted that evident contrast enhancement of an anterior mediastinal mass does not necessarily indicate a TET, and it is necessary to consider other soft-tissue lesions such as thymic hyperplasia (19), germ cell tumor (20), or lymphoma (21) in young adults, although those lesions tend to be around 5 $\mathrm{cm}$ or larger.

Not surprisingly, protrusion was a characteristic morphological CT finding of even small TETs $(52.9 \%$ in TETs sized 1-2 cm). Because TETs are neoplasms, they can protrude easily without being confined by low-tension structures such as the lung and mediastinal pleura. In contrast, cysts do not easily protrude from the mediastinal pleura because of their high compressibility. If a cyst shows protrusion, it tends to protrude in a wide and broad-based shape, leading to a low protrusion ratio of up to $10 \%$. In addition, TETs are more frequently found in off-midline locations than cysts $(7,22)$, which likely contributes to the high protrusion ratio of even small TETs.

Several studies have shown that ${ }^{18} \mathrm{~F}$-FDG PET uptake varies across the histological subgroups of TETs, and it is also 
known to be useful for distinguishing between benign and malignant lesions $(8,9,23,24)$. Shinya et al. (9) showed that the diagnostic value of ${ }^{18} \mathrm{~F}-\mathrm{FDG}$ PET/CT is comparable to CT for predicting thymic carcinoma ( $A U C=0.855$ vs. 0.853 ). Tatci et al. (24) showed that SUVmax was significantly higher in solid benign lesions than in cystic benign lesions, but these authors included heterogeneous groups of benign solid masses in their evaluation. Our study found that even small, low-grade thymomas showed significantly higher SUVmax values than thymic cysts. Accordingly, ${ }^{18} \mathrm{~F}-\mathrm{FDG}$ PET/ CT scans can reliably discriminate small cystic lesions from TETs in the anterior mediastinum, although the radiation exposure of PET/CT scans is a concern (25).

Magnetic resonance imaging (MRI) is another imaging modality useful for the characterization of anterior mediastinal lesions $(26,27)$. Cysts typically show high signal intensity on T2-weighted images; thus, they may be easily distinguished from solid tumors even in cases with hemorrhage or inflammation that cause high attenuation in CT. Tomiyama et al. (4) reported that correct diagnosis rates for CT and MRI for thymic cysts were $46.0 \%$ and $71.0 \%$ ( $p$ $<0.05)$, respectively. Nevertheless, accessibility to MRI is limited for some institutions due to high cost and long acquisition times and occasionally MRI requires considerable breath-holding; therefore, $\mathrm{CT}$ would be the practical choice for less cooperative patients $(26,28)$. There is a consensus that chest CT is a reliable, cost-effective, and appropriate diagnostic tool for thymic lesions according to the European Society of Thoracic Surgeons (29).

Our study has several limitations. First, the study population was retrospectively recruited and various CT scanners were used, although contrast-enhanced CT scans were performed using a standardized institutional protocol. Second, the benign cyst group included the most prevalent cysts in the mediastinum, both bronchogenic cysts and thymic cysts, and we did not analyze the two subgroups separately. In addition, we could not include pericardial cysts in the anterior mediastinum due to paucity of the pathologically proven cysts, which can be correctly diagnosed on CT by water attenuation in most cases (3). Third, we only included TETs in the solid tumor group. Other solid neoplasms can arise from the anterior mediastinum, including germ cell tumors, lymphomas, and neuroendocrine tumors $(4,7,30,31)$. However, most of those neoplasms occur in young adults and are usually found symptomatically, whereas incidental anterior mediastinal lesions on screening chest $\mathrm{CT}$ in the elderly were mostly TETs and cysts $(1,2)$. Finally, the validation set was relatively small. An additional validation study is required with a larger patient population.

In conclusion, the $\mathrm{CT}$ features of post-contrast attenuation $>60 \mathrm{HU}$ and protrusion are useful imaging parameters for distinguishing TETs from cysts in the anterior mediastinum. SUVmax on ${ }^{18} \mathrm{~F}$-FDG PET scans can also be a reliable tool for discriminating these lesions. Unnecessary thymectomy for benign cysts in the anterior mediastinum can be avoided with the help of these CT and ${ }^{18} \mathrm{~F}$-FDG PET findings.

\section{Supplementary Materials}

The online-only Data Supplement is available with this article at https://doi.org/10.3348/kjr.2018.0400.

\section{Conflicts of Interest}

The authors have no potential conflicts of interest to disclose.

ORCID iDs

Soon Ho Yoon

https://orcid.org/0000-0002-3700-0165

Sang Hyup Lee

https://orcid.org/0000-0002-6773-2965

Ju Gang Nam

https://orcid.org/0000-0003-3991-4523

Hyung Jin Kim

https://orcid.org/0000-0003-0722-0033

Su Yeon Ahn

https://orcid.org/0000-0002-3370-0498

Hee Kyung Kim

https://orcid.org/0000-0001-7400-1311

Hyun Ju Lee

https://orcid.org/0000-0001-5748-2096

Gi Jeong Cheon

https://orcid.org/0000-0002-1360-5186

Jin Mo Goo

https://orcid.org/0000-0003-1791-7942

\section{REFERENCES}

1. Henschke CI, Lee IJ, Wu N, Farooqi A, Khan A, Yankelevitz D, et al. CT screening for lung cancer: prevalence and incidence of mediastinal masses. Radiology 2006;239:586-590

2. Yoon SH, Choi SH, Kang CH, Goo JM. Incidental anterior 
mediastinal nodular lesions on chest CT in asymptomatic subjects. J Thorac Oncol 2018;13:359-366

3. Jeung MY, Gasser B, Gangi A, Bogorin A, Charneau D, Wihlm $\mathrm{JM}$, et al. Imaging of cystic masses of the mediastinum. Radiographics 2002;22 Spec No:S79-S93

4. Tomiyama N, Honda 0, Tsubamoto M, Inoue A, Sumikawa H, Kuriyama K, et al. Anterior mediastinal tumors: diagnostic accuracy of CT and MRI. Eur J Radiol 2009;69:280-288

5. Araki T, Sholl LM, Gerbaudo VH, Hatabu H, Nishino M. Intrathymic cyst: clinical and radiological features in surgically resected cases. Clin Radiol 2014;69:732-738

6. Jurado J, Javidfar J, Newmark A, Lavelle M, Bacchetta M, Gorenstein $\mathrm{L}$, et al. Minimally invasive thymectomy and open thymectomy: outcome analysis of 263 patients. Ann Thorac Surg 2012;94:974-981; discussion 981-982

7. Ackman JB, Verzosa S, Kovach AE, Louissaint A Jr, Lanuti M, Wright $C D$, et al. High rate of unnecessary thymectomy and its cause. Can computed tomography distinguish thymoma, lymphoma, thymic hyperplasia, and thymic cysts? Eur J Radiol 2015;84:524-533

8. Lococo F. The role of $18 \mathrm{~F}-\mathrm{FDG}$ PET/CT scan in predicting histological type of thymic epithelial tumors: indications and practical clinical considerations. Thorac Cardiovasc Surg 2018;66:350-351

9. Shinya T, Tanaka T, Soh J, Matsushita T, Sato S, Toyooka S, et al. Diagnostic value of dual-time-point F-18 FDG PET/CT and chest CT for the prediction of thymic epithelial neoplasms. Acta Med Okayama 2017;71:105-112

10. Choi YW, McAdams HP, Jeon SC, Hong EK, Kim YH, Im JG, et al. Idiopathic multilocular thymic cyst: CT features with clinical and histopathologic correlation. AJR Am J Roentgenol 2001;177:881-885

11. Maki DD, Birnbaum BA, Chakraborty DP, Jacobs JE, Carvalho BM, Herman GT. Renal cyst pseudoenhancement: beamhardening effects on CT numbers. Radiology 1999;213:468472

12. Mileto A, Nelson RC, Samei E, Jaffe TA, Paulson EK, Barina A, et al. Impact of dual-energy multi-detector row $\mathrm{CT}$ with virtual monochromatic imaging on renal cyst pseudoenhancement: in vitro and in vivo study. Radiology 2014;272:767-776

13. Chen G, Marx A, Chen WH, Yong J, Puppe B, Stroebel P, et al. New WHO histologic classification predicts prognosis of thymic epithelial tumors: a clinicopathologic study of 200 thymoma cases from China. Cancer 2002;95:420-429

14. Nishino M, Ashiku SK, Kocher ON, Thurer RL, Boiselle PM, Hatabu H. The thymus: a comprehensive review. Radiographics $2006 ; 26: 335-348$

15. Wang X, Chen K, Li X, Li Y, Yang F, Li J, et al. Clinical features, diagnosis and thoracoscopic surgical treatment of thymic cysts. J Thorac Dis 2017;9:5203-5211

16. Bae KT, Heiken JP, Siegel CL, Bennett HF. Renal cysts: is attenuation artifactually increased on contrast-enhanced CT images? Radiology 2000;216:792-796
17. Birnbaum BA, Hindman N, Lee J, Babb JS. Renal cyst pseudoenhancement: influence of multidetector CT reconstruction algorithm and scanner type in phantom model. Radiology 2007;244:767-775

18. Wang ZJ, Coakley FV, Fu Y, Joe BN, Prevrhal S, Landeras LA, et al. Renal cyst pseudoenhancement at multidetector CT: what are the effects of number of detectors and peak tube voltage? Radiology 2008;248:910-916

19. Araki T, Sholl LM, Gerbaudo VH, Hatabu H, Nishino M. Imaging characteristics of pathologically proven thymic hyperplasia: identifying features that can differentiate true from lymphoid hyperplasia. AJR Am J Roentgenol 2014;202:471-478

20. Levitt RG, Husband JE, Glazer HS. CT of primary germcell tumors of the mediastinum. AJR Am J Roentgenol 1984;142:73-78

21. Tateishi U, Muller NL, Johkoh T, Onishi Y, Arai Y, Satake M, et al. Primary mediastinal lymphoma: characteristic features of the various histological subtypes on CT. J Comput Assist Tomogr 2004;28:782-789

22. McErlean A, Huang J, Zabor EC, Moskowitz CS, Ginsberg MS. Distinguishing benign thymic lesions from early-stage thymic malignancies on computed tomography. J Thorac Oncol 2013;8:967-973

23. Sung YM, Lee KS, Kim BT, Choi JY, Shim YM, Yi CA. 18F-FDG PET/CT of thymic epithelial tumors: usefulness for distinguishing and staging tumor subgroups. J Nucl Med 2006;47:1628-1634

24. Tatci E, Ozmen 0, Dadali Y, Biner IU, Gokcek A, Demirag F, et al. The role of FDG PET/CT in evaluation of mediastinal masses and neurogenic tumors of chest wall. Int J Clin Exp Med 2015;8:11146-11152

25. Huang B, Law MW, Khong PL. Whole-body PET/CT scanning: estimation of radiation dose and cancer risk. Radiology 2009;251:166-174

26. Daye D, Ackman JB. Characterization of mediastinal masses by MRI: techniques and applications. Appl Radiol 2017;46:1022

27. Zhang W, Zhou Y, Xu XQ, Kong LY, Xu H, Yu TF, et al. A wholetumor histogram analysis of apparent diffusion coefficient maps for differentiating thymic carcinoma from lymphoma. Korean J Radiol 2018;19:358-365

28. Ackman JB, Wu CC. MRI of the thymus. AJR Am J Roentgenol 2011;197:W15-W20

29. Ruffini E, Van Raemdonck D, Detterbeck F, Rocco G, Thomas P, Venuta F. Management of thymic tumors: a survey of current practice among members of the European Society of Thoracic Surgeons. J Thorac Oncol 2011;6:614-623

30. Takahashi K, Al-Janabi NJ. Computed tomography and magnetic resonance imaging of mediastinal tumors. J Magn Reson Imaging 2010;32:1325-1339

31. Shahrzad M, Le TS, Silva M, Bankier AA, Eisenberg RL. Anterior mediastinal masses. AJR Am J Roentgenol 2014;203:W128-W138 